\title{
Analizando las posibles aplicaciones de los drones y los satélites en la enseñanza de Fisiología Vegetal
}

Vanessa Martos Núñez - Universidad de Granada
Ali Ahmad - Universidad de Granada
Belén García del Moral Garrido - Universidad de Almería

Recepción: 27.12.2022 | Aceptado: 25.01.2022

Correspondencia a través de ORCID: Vanessa Martos
(iD) $0000-0001-6442-7968$

(D) $0000-0001-5530-7374$

(D) 0000-0001-9803-9939

Citar: Martos Núñez, V, Ahmad, A y García del Moral Garrido, B (2022). Analizando las posibles aplicaciones de los drones y los satélites en la enseñanza de Fisiología Vegetal. REIDOCREA, 11(8), 90-93.

Estudio de investigación desarrollado gracias a los proyectos FEDER-Junta de Andalucía: VIRTUOUS-P184700, proyecto UE-H2020-RISE-2020-SUSTAINABLE ref. 101007702 y EU-H2020-RISE-2019-VIRTUOUS ref. 872181

\section{Área o categoría del conocimiento: Didáctica de la Agricultura}

Resumen: El año 2021 ha sido marcado oficialmente como el inicio de la era de la "Industria 5.0" por la Comisión Europea. Esta quinta revolución tecnológica puede revolucionar la industria agrícola al a) facilitar la integración de nuevas tecnologías b) mejorar la eficiencia de las operaciones agrícolas. En este sentido, los drones y los satélites son dos de las muchas tecnologías previstas, ya que están allanando el camino hacia una agricultura sostenible y de precisión: añadiendo así un valor sustancial a la adquisición de datos de los cultivos, ideando simulaciones de rendimiento, prediciendo los períodos de cosecha y de riego, erradicando los obstáculos de los datos metrológicos, etc. Las continuas mejoras técnicas y pragmáticas de ambas, a lo largo de las tres últimas décadas, han contribuido considerablemente a la madurez de estas tecnologías, anticipando así su eficaz integración en la agricultura. Sus aplicaciones se resumen en la tabla 1. Dada la gran importancia de estas tecnologías novedosas y proactivas, es indispensable educar y reforzar a los estudiantes para que aprovechen al máximo el potencial de la agricultura actual, garantizando así la sostenibilidad.

\section{Palabra clave: Agricultura 4.0}

\section{Looking into the potential applications of drones and satellites in plant physiology teaching}

Abstract: The year 2021 has been officially marked as the beginning of the era of "Industry 5.0" by the European Commission. This 5th technological revolution can revolutionize the agricultural industry by a) facilitating the integration of novel technologies $b$ ) enhancing the efficiency of agricultural operations. In this regard, drones and satellites are two of the many anticipated technologies, as they are paving the way towards a sustainable and precision farming: thereby adding a substantial value to crop data acquisition, devising yield simulations, predicting the harvesting and irrigation periods, eradicating the metrological data hindrances etc. The continuous technical and pragmatic improvements of the two, over the past three decades, have considerably contributed towards the maturity of these technologies, thus anticipating their efficient integration into agriculture. Their applications are summarized in Table 1. Given the significant importance of these sustainably proactive and novel technologies, it is indispensable to educate and reinforce the students, to maximize the potential of today's agriculture: thereby ensuring sustainability

Keyword: Agriculture 4.0

\section{Introducción}

El principal componente de los drones y los satélites que es vital para la adquisición de datos es el sensor de a bordo. Los sensores proporcionan toda la información básica sobre factores como la temperatura, la humedad, las enfermedades, la luz y el nivel de dióxido de carbono (CO2), que son vitales para el crecimiento y el rendimiento de los cultivos. Estos factores, junto con otros muchos parámetros, pueden medirse fácilmente con sensores (Ahmad et al. 2021). 
Los sensores miden las radiaciones electromagnéticas reflejadas o emitidas por el objeto de estudio. Los sensores utilizados para la vigilancia de los cultivos detectan la banda infrarroja térmica, las bandas roja, verde y azul (RGB), la banda infrarroja cercana (NIR) y la banda de borde rojo (RE) de las ondas electromagnéticas. En consecuencia, pueden clasificarse según las radiaciones que registran en pasivos (cámaras, escáneres, etc.) y activos (radar y LIDAR). En la actualidad, los sensores más utilizados en la agricultura incluyen el radar de apertura sintética (SAR), el infrarrojo cercano (NIR), la detección y el alcance de la luz (LiDAR), la espectroscopia de fluorescencia y la obtención de imágenes, los sensores multiespectrales y los visibles RGB (VIS), con lo que se estudian diversos parámetros según las necesidades (Tabla 1).

Tabla 1. Varios sensores utilizados en agricultura con sus respectivas aplicaciones.

\begin{tabular}{ll}
\hline Sensor & Aplicación \\
\hline $\begin{array}{l}\text { Apertura Sintética Radar } \\
\text { (SAR) }\end{array}$ & $\begin{array}{l}\text { Clasificación de cultivos, control del crecimiento de los cultivos y control de la humedad del } \\
\text { suelo. } \\
\text { Visible RGB (VIS) } \\
\begin{array}{l}\text { Multiespectral e } \\
\text { hiperespectral } \\
\text { Espectroscopía de }\end{array} \\
\begin{array}{l}\text { Fluorescencia } \\
\text { Atributos fisiológicos y bioquímicos (índice de área foliar, contenido de agua de los cultivos, } \\
\text { contenido de clorofila de las hojas/dosel y contenido de nitrógeno). } \\
\text { de luz (LiDAR) }\end{array} \\
\text { Contenido de clorofila y nitrógeno, relación nitrógeno/carbono e índice de área foliar. } \\
\text { Infrarojo cercano (NIR) }\end{array}$ \\
\hline
\end{tabular}

\section{Objetivos}

El objetivo general de este estudio consiste en implementar en las enseñanzas de Grado y Máster de áreas relacionadas con la Agronomía, la formación en la toma de datos de las principales plataformas utilizadas actualmente en agricultura.

Existen los siguientes pasos generales para utilizar las tecnologías de drones y satélites en las operaciones agrícolas, aunque depende en gran medida del propósito y la necesidad del estudio.

Los objetivos específicos son:

1. Adquisición de datos, a través de sensores.

2. Preprocesamiento de los datos adquiridos: clasificación, organización, etiquetado, etc.

3. Procesamiento: construcción de ortomosaicos, síntesis de índices de vegetación, etc.

4. Interpretación de los resultados y medidas subsiguientes; por ejemplo, regar las plantas con falta de agua.

\section{Método}

El método se basa en el cálculo de los índices, Una información importante sobre la fisiología de los cultivos se obtiene a través de los índices de vegetación (IV), que son las combinaciones de reflectancia de la superficie en una determinada longitud de onda, normalmente dos o más, destinadas a proporcionar información sobre la vegetación. Por ejemplo, el índice de clorofila - verde (Cl-G) de la vegetación pone de manifiesto la capacidad fotosintética de la planta cuantificando la clorofila por unidad de superficie. Existen varios IV, cada uno de ellos con una finalidad determinada (Tabla 2). Uno de los 
IV más comunes es el índice de vegetación de diferencia normalizada (NDVI), que proporciona información fisiológica sobre la salud de los cultivos, es decir, la producción de biomasa, la cobertura y densidad del dosel, la detección del estrés hídrico, etc.

Tabla 2. Índices de vegetación (IV).

\begin{tabular}{|c|c|c|}
\hline Nombre & Función & Ecuación \\
\hline $\begin{array}{l}\text { Índice de Diferencia Normalizada de } \\
\text { Vegetación (NDVI) }\end{array}$ & $\begin{array}{l}\text { Monitorización de los cultivos y estudios } \\
\text { empíricos. }\end{array}$ & $\frac{\mathrm{NIR}-\mathrm{R}_{\text {red }}}{\mathrm{NIR}+\mathrm{R}_{\text {red }}}$ \\
\hline $\begin{array}{l}\text { Índice de Vegetación Ajustado al Suelo } \\
\text { (SAVI) }\end{array}$ & $\begin{array}{l}\text { Mejora de la sensibilidad del NDVI a los } \\
\text { fondos del suelo. }\end{array}$ & $\frac{(1+0.5)\left(\mathrm{NIR}-\mathrm{R}_{\mathrm{red}}\right)}{\mathrm{NIR}+\mathrm{R}_{\mathrm{red}}+0.5}$ \\
\hline Índice de Vegetación Clorofílica (CVI) & $\begin{array}{l}\text { Representación de la abundancia relativa } \\
\text { de la vegetación y el suelo. }\end{array}$ & $\mathrm{NIR} * \frac{\mathrm{R}_{\text {red }}}{\mathrm{R}_{\text {green }}^{2}}$ \\
\hline $\begin{array}{l}\text { Índice de Vegetación de Diferencia } \\
\text { Normalizada de Vegetación (gNDVI) }\end{array}$ & Estimación de la actividad fotosintética. & $\frac{\mathrm{NIR}-\mathrm{R}_{\text {green }}}{\mathrm{NIR}+\mathrm{R}_{\text {green }}}$ \\
\hline Índice de clorofila - verde (Cl-G) & $\begin{array}{l}\text { Determinación del contenido de clorofila } \\
\text { en la hoja. }\end{array}$ & $\frac{\mathrm{NIR}}{\mathrm{R}_{\text {green }}}-1$ \\
\hline $\begin{array}{l}\text { Índice de Diferencia verde-rojo } \\
\text { Normalizado (NGRDI) }\end{array}$ & Estimación del estado nutritivo & $\frac{R_{\text {green }}-R_{\text {red }}}{R_{\text {green }}+R_{\text {red }}}$ \\
\hline Índice de Hoja Verde (GLI) & Estimación del contenido en clorofila. & $\frac{2 \cdot R_{\text {green }}-R_{\text {red }}-R_{\text {blue }}}{2 \cdot R_{\text {green }}+R_{\text {red }}+R_{\text {blue }}}$ \\
\hline $\begin{array}{l}\text { Índice de Estrés hídrico de los Cultivos } \\
\text { (CWSI) }\end{array}$ & $\begin{array}{l}\text { Medición de los cambios y la dinámica de } \\
\text { la temperatura del dosel. }\end{array}$ & $\frac{\mathrm{T}_{\text {canopy }}-\mathrm{T}_{\text {nws }}}{\mathrm{T}_{\text {dry }}-\mathrm{T}_{\text {nws }}}$ \\
\hline $\begin{array}{l}\text { Índice Fotoquímico de Reflectancia } \\
\text { (PRI) }\end{array}$ & Detección de síntomas de enfermedades. & $\frac{R_{531}-R_{570}}{R_{531}-R_{570}}$ \\
\hline \multicolumn{3}{|c|}{$\begin{array}{l}\text { Donde: NIR es la reflectancia de la banda del infrarrojo cercano, Rred es la reflectancia de la banda del rojo; Rblue es la reflectancia de la banda } \\
\text { del azul; Rgreen es la reflectancia de la banda del verde; Tcanopy es la temperatura del dosel de las hojas bajo la luz del sol; Tnws es la } \\
\text { temperatura del dosel de las hojas bajo la luz del sol cuando el cultivo está bien regado; Tdry es la temperatura del dosel de las hojas bajo la } \\
\text { luz del sol cuando el cultivo está bajo estrés por sequía. }\end{array}$} \\
\hline
\end{tabular}

\section{Discusión}

\section{Drones vs. Satélites}

Ambos tienen sus propias ventajas y desventajas. Por ejemplo, varios organismos gubernamentales, como la Unión Europea, la Administración Nacional de Aeronáutica y del Espacio (NASA), etc., ofrecen gratuitamente imágenes por satélite con una importante resolución espacial y espectral. Por otro lado, características atractivas como la flexibilidad del tiempo de vuelo, la maniobrabilidad y las ventajas económicas hacen que los drones sean superiores a la tecnología satelital, pero ambos pueden considerarse como alternativas, y son indispensables para lograr una agricultura sostenible (Khanal et al. 2020). Asimismo, ambos sirven para desarrollar índices de vegetación (IVs) que proporcionan información relacionada con el estrés, las enfermedades y el crecimiento del cultivo. Algunos de los IVs comúnmente calculados, entre otros, incluyen el índice de vegetación de diferencia normalizada (NDVI), el índice de vegetación ajustado al suelo (SAVI), el índice de clorofila - verde (Cl-G), el índice de diferencia verde-rojo normalizado (NGRDI), y el índice de reflectancia fotoquímica (PRI), etc. De todos ellos, el NDVI proporciona información fisiológica sobre la salud de los cultivos, es decir, la producción de biomasa, la cobertura y la densidad del dosel, la detección del estrés hídrico, etc. 
La batería y el tiempo de vuelo limitados dificultan la amplia cobertura espacial de los RPA, a pesar de que se consideran óptimos para proporcionar una fenotipificación de los cultivos robusta, fiable y eficiente. Sin embargo, los RPAs ofrecen menores costes operativos pero para grandes datos (para cubrir áreas más extensas) los costes de procesamiento de datos aumentan exponencialmente. Del mismo modo, la flexibilidad con los tiempos de vuelo y una resolución significativa de las imágenes RPA son las características deseadas sobre las imágenes de satélite.

Por otro lado, los datos satelitales de libre acceso están ganando popularidad. Aun así, estos datos de libre acceso vienen con una resolución espacial y temporal más gruesa (con la excepción de unos pocos satélites comerciales), y se están utilizando para generar Vis (Unal et al. 2014) En este sentido, la IA ofrece la posibilidad de afinar los datos satelitales más gruesos basándose en los datos de los drones de alta resolución. Además, un gran conjunto de datos, a pesar de su baja calidad o ruido, para el entrenamiento de modelos de IA genera resultados sustanciales en comparación con conjuntos de datos más pequeños, lo que indica la importancia de los enormes conjuntos de datos obtenidos mediante imágenes de satélite (Saiz-Rubio et al. 2020).

\section{Conclusión}

Es necesario un conocimiento básico de los últimos avances en tecnologías emergentes y de los diferentes IVs así como de las diferentes bases de datos de acceso abierto para la recogida de datos por satélite aplicados en Fisiología Vegetal. Dada la gran importancia de estas tecnologías novedosas y proactivas, es indispensable educar y reforzar a los estudiantes, para maximizar el potencial de la agricultura actual: garantizando así la sostenibilidad.

\section{Referencias}

Ahmad, A, Ordoñez, J, Cartujo, P, \& Martos, V (2021). Remotely Piloted Aircraft (RPA) in Agriculture: A Pursuit of Sustainability. Agronomy, 11(1), 7. https://doi.org/10.3390/agronomy11010007

Unal, I, \& Topakci, M (2014). A review on using drones for precision farming applications. In Proceedings of the 12th International Congress on Agricultural Mechanization and Energy, Cappadocia, Turkey (pp. 3-6).
Khanal, S, KC, K, Fulton, JP, Shearer, S, \& Ozkan, E (2020). Remote sensing in agriculture-accomplishments, limitations, and opportunities. Remote Sensing, 12(22), 3783. https://doi.org/10.3390/rs12223783

Saiz-Rubio, V, \& Rovira-Más, F (2020). From smart farming towards agriculture 5.0: A review on crop data management. agronomy, 10(2), 207. https://doi.org/10.3390/agronomy 10020207 